\section{Rocky Mountain ACP/AMA Internal Medicine Conference}

\author{
November 14-16, 2019
}

Research Abstracts

\section{A ROCKY MOUNTAIN
Internal Medicine}

\section{General Internal Medicine Fellows as Teachers in the Ambulatory Clinic}

Cary Cuncic, MD, FRCP(C), MMEd, MSc., James Tessaro, $M D, F R C P(C), M H P E$, Harpinder Nagi, MD, FRCP $(C)$, Aman Nijjar, MD, FRCP(C), $M P H$

Department of Medicine, University of British Columbia

\section{Background}

Recruitment and retention of preceptors in medical student ambulatory clinic is challenging; reasons cited include hospital based medical teaching units being more familiar and a lack of remuneration. As a solution, we assigned our General Internal Medicine (GIM) fellows to teach in clinic, in hoping to decrease the teaching load and serve as early faculty development.

\section{Methods}

We assigned medical students to GIM fellows during their longitudinal clinic. Fellows were responsible for the teaching and assessment of the students. We interviewed attending physicians, fellows and students about their experiences in this model. Using a grounded theory approach to both data collection and analysis and subsequent conversations with a key informant, we identified emergent themes and best practices.

\section{Results}

The model was successful with students and fellows. The students felt that the learning environment was supportive, the fellow was a positive role model, and that they received good quality teaching and feedback. The fellows felt that the students' enthusiasm was contagious, that they learned different skills as a clinic "junior attending", and many agreed that they would continue to teach in clinic. Attending physicians had a mixed view. Those who had not taught students prior felt ill - prepared. Some questioned the fellows' educational experience. All agreed that the current model was untenable, due to the lack of organization.

\section{Conclusions}

Having GIM fellows as teachers in the internal medicine ambulatory clinic is pedagogically sound, as it offers benefits to both sets of learners. In addition, faculty development is occurring at the level of the fellow, so as to recruit future clinical teachers. However the operationalization was unsuccessful due to the chaotic nature. We propose adding a GIM fellow as a "junior attending" to the blocked medical student clinic to help ameliorate the identified challenges.

\section{To Evaluate and Compare the Expectations and} Attitude Towards Precision Medicine Among the Patients and Physicians in Oncology

Dr. Winson Cheung ${ }^{1}$, Dr. Gwen Bebb ${ }^{2}$, Dr Navdeep Dehar ${ }^{3 * * *}$

Department of Medical Oncology, TBCC $1,{ }^{2}$, Department of Internal Medicine, University of Calgary ${ }^{3}$

\section{Background}

Despite the potential benefits of genomic testing in cancer diagnosis and treatment, this technology is relatively new to most cancer patients. Prior research has reported insufficient understanding of genomic testing, as well as apprehensiveness towards the potentially overwhelming test results. However, there has been very few studies of patient knowledge and expectations of genomic testing and it remains unclear how the perspectives of patient and physician differ in regards to the use of this new technology. Such discrepancies in patient and physician knowledge and expectations can often result in suboptimal cancer management and follow-up care. We propose a survey study that aims to examine the potential differences patient and physician views towards genomic testing in cancer. 


\section{Objective}

To evaluate and compare the expectations and attitude towards precision medicine among the patients and physicians in Oncology

\section{Research Methodology}

This study will be conducted in Oncology clinics at the TBCC. The participating oncologists will be asked to complete the physician survey and approach patients they are diagnosing and treating and who meet the inclusion criteria. Interested patients will be asked complete the survey questionnaire in paper format. Two separate but complementary self-administered questionnaire survey have been developed and will be distributed for a period of 1-2 years. Survey responses from patients will be matched with those of their oncologists to form patient-oncologist pairs, respectively.

\section{Statistical Analysis}

Multivariable logistic regression analyses will be conducted to calculate the odds ratios for discordant genomic testing expectations, dependent on patient factors and physician characteristics. All tests will be two-sided, where a P value less than .05 will be considered statistically significant.

\section{Conclusion}

Identifying the areas of agreement and disagreement between patients and providers can assist the development of strategies to improve education and counseling of genomic testing, which can in turn enhance its use and optimize patient-specific cancer management.

\section{Determining Patient and Provider Variables that Contribute to the Selection of In-Hospital Diabetes Regimen in Patients Admitted to Acute Care Hospitals}

Erin Helson (MD), Evan Hagen (BSc), Karmon Helmle $(M S c, M D)$, Edwin Rogers (Senior analyst), and

Shannon Ruzycki (MPH, MD)

Department of Medicine, University of Calgary

\section{Background}

Basal bolus insulin therapy (BBIT) is recommended over sliding scale (SS) regimens for patients with diabetes admitted to the hospital. Despite this, many patients are placed on SS. The patient or clinical characteristics that are associated with selection of BBIT or SS are not known. The aim of this study is to determine patient and provider factors that influence in-hospital glycemic management regimens.

\section{Methods}

We performed a retrospective cross-sectional study of adult patients with diabetes admitted to internal medicine for greater than 48 hours between April 1st, 2016 and July 31st, 2018 in Calgary, Alberta. We included patients with a HbAlc result within six months prior to admission, and who received insulin during admission. Patients admitted from long term care, admitted to intensive care, who were pregnant, who received a blood transfusion, who had type 1 diabetes, or had palliative goals of care were excluded.

\section{Results}

Eight-hundred and three patients were included; 469 [58\%] were on BBIT and 334 [42\%] were on SS. Compared to those on SS, BBIT patients were younger ( 61.5 vs. $68.6 ; \mathrm{p}<0.001)$, less often at target $\mathrm{HbA1C}(52 \%$ versus $18 \%$; $\mathrm{p}<0.001)$, and more likely to use insulin prior to admission ( $92 \%$ vs $10 \%$; $p<0.001)$. BBIT patients had higher mean glucose in the first 48 hours of admission compared to those on SS (10.9 mM [3.69] versus 8.9 $\mathrm{mM}$ [2.32]; $\mathrm{p}<0.001)$. A greater proportion of BBIT patients had hypoglycemic events ( $26 \%$ vs $5 \% ; \mathrm{p}<0.001)$ and severe hyperglycemic events ( $35 \%$ vs $6 \%$; $\mathrm{p}<0.001$ ).

\section{Conclusion}

These results suggest that there may be important differences between patients who are started on BBIT and those started on SS. Any non-randomized study that compares outcomes between these regimens may be susceptible to important confounding.

\section{Retrospective Study of Patients with Upper Extremity Clots Presenting to Emergency Departments in Tertiary Care Hospitals in Calgary in the Last Five Years.}

Tania Pannu, $M D^{1 \star}$, Eric Herget, MD1 and

Deepa Suryanarayan, $M B B S^{1}$

${ }^{1}$ University of Calgary, Calgary, AB, Canada

\section{Objective}

5 year retrospective study of patients presenting to emergency departments at the four tertiary care hospitals in Calgary with upper extremity (UE) deep vein thrombosis (DVT) to determine practice patterns and management of UE clots.

\section{Methods}

Patients with suspected UE DVT with diagnostic imaging from January 2014 to December 2018 were included in the study. Inclusion criteria included age above 18 years. Exclusion criteria were any known 
cancer or life expectancy under 6 months. Basic demographic data were collected, in addition to disposition from emergency, choice of anticoagulant, duration of anticoagulation, history of thrombophilia and thoracic outlet obstruction (TOO) work-up.

\section{Results}

1236 patient records were reviewed, of which $12.2 \%$ were positive for UE DVT and 9.2\% for superficial vein thrombosis (SVT). Mean age was 47 years and $50.2 \%$ were males. Mean duration of treatment for DVT and SVT was 5.01 months and 1.15 months, respectively. $22.5 \%$ of all DVTs were recommended lifelong therapy. 21 (18.4\%) patients were treated for more than 45 days for SVT, of which only 9 had another indication for anticoagulation. Line associated thrombosis was the most common risk factor identified (17.2\%). Anticoagulant agents for DVT included low molecular weight heparin (7.9\%), direct oral anticoagulants (39.1\%) and warfarin $(42.4 \%) .8 .6 \%$ of the DVTs received no treatment. $30.5 \%$ of the patients with DVT were referred to General Internal Medicine, $24.5 \%$ to Hematology and $33.7 \%$ to their family doctor. $39.7 \%$ of the people with DVT were tested for thrombophilia, of which $10 \%$ tested positive for any type of thrombophilia. $29.8 \%$ were tested for TOO, of which $48.9 \%$ were positive and $11.1 \%$ received surgery for same.

\section{Conclusion}

Our study demonstrates significant variability in the management of UE clots. Next steps include developing a structured, standardized approach to help reduce this variability and optimize medical management.

\section{Using a TRIZ to Understand the Current Challenges and Potential Solutions in the Medical Teaching Unit}

Pirani F, MD; Altabbaa G, MD, MSc;

Grinman $M, M D, M P H$

\section{Background}

The modern Medical Teaching Unit (MTU) is confronted with the challenge of balancing increasing patient complexity, increasing workload \& limited resources, with the need to provide safe \& cost-effective care in an optimum teaching environment for trainees that maintains physician wellbeing.

\section{Aim \\ To better understand the MTU environment at our hospital, we designed a TRIZ to elicit current challenges/potential solutions as perceived by various healthcare providers.}

\section{Improvement/Innovation}

We conducted 3 sessions - two with residents/medical students, and another with allied health staff. These results are being used to design a quality-improvement initiative in the upcoming year. When asked to "describe the worst possible MTU" participants generated a theoretical list of problems. They were then asked to identify which of these are currently happening, and suggest potential solutions. Subsequently, participants prioritized the problem/ solution list according to ease of implementation \& potential impact. A fishbone diagram was created for thematic analysis. Among the ten major issues identified, the most common themes were a lack of communication (oral/written) between team members and organizational structure of daily activities, both of which have easily implementable solutions with relatively high potential impact.

\section{Measures}

When a change initiative is implemented, we will select measures to determine if we are achieving the Quadruple Aim - improved patient/provider satisfaction (eg: surveys), healthcare resource utilization (eg: length of stay) \& patient outcomes (eg: complication rate).

\section{Next Steps}

Our results have implications for changing several processes, many of which impact attending physicians. Therefore, we will perform a TRIZ with the faculty next to inform the design \& implementation of a change initiative directed toward improving the quadruple aim on the MTU.

\section{Impact}

We expect this study will enable us to better understand the needs of different healthcare providers through a systemsthinking approach that optimizes operations striving to achieve the Quadruple Aim.

\section{Identifying Learning Needs in Medical Assistance in Dying of Postgraduate Medical Residents}

\author{
Dr. Krista Reich, Dr. Jacqueline Hui, Dr. Amy Tan
}

University of Calgary

Medical Assistance in Dying (MAiD) is now legal in Canada. Therefore, integrating MAiD into the medical curriculum will be important for providing trainees with the skills, knowledge, and attitudes to care for patients requesting assistance in dying. To date, there is no literature available on educational content and format of MAiD teaching. The objective of this study was to determine 
the learning needs in MAiD of postgraduate Internal Medicine (IM) residents.

A learning needs assessment was completed during an IM academic half day at the University of Calgary. Demographic information and data on prior teaching in MAiD was obtained before the session. Residents were then taken through three cases, independently online, and then as a group facilitated by an expert in MAiD content. The cases were designed to test situational judgement and resident knowledge in MAiD. A thematic analysis was performed to identify learner gaps in knowledge.

Demographic data was obtained from 20 participants, all IM residents, of which $65 \%$ were female and $50 \%$ were in their first year of residency. A total of $40 \%(8 / 20)$ of the residents received MAiD teaching before residency, with an average of $3.75 \pm 1.5$ hours. In assessing confidence in responding to a MAiD request, 74\% (14/19) of residents reported to be "not very confident", and $100 \%$ of residents (20/20) thought more education in MAiD should be incorporated into their residency training. High level themes included "resident discomfort with discussing MAiD, lack of understanding of the local MAiD policy and procedures, and perceptions that MAiD is only an option when symptoms are optimized".

Preliminary results demonstrate gaps of knowledge in eligibility, policy and procedures, including referral process, and understanding the role and obligations of residents in the request process. Overall, there is a need for more residency training in this specialized area.

\section{An Alternative Prophylaxis for Deep Vein Thrombosis Using Intermittent Electrical Stimulation}

\section{Kahir A Rahemtulla ${ }^{1,3}$, Dirk G Everaert ${ }^{1,3}$, Michel JA Gauthier, Vivian K Mushahwar ${ }^{1,2,3}$}

${ }^{1}$ Department of Medicine, Faculty of Medicine and Dentistry, University of Alberta, Canada

${ }^{2}$ Neuroscience and Mental Health Institute, University of Alberta, Canada

${ }^{3}$ Sensory Motor Adaptive Rehabilitation Technology

(SMART) Network, University of Alberta, Canada

kahir@ualberta.ca

Funding support received: Glenrose Hospital Foundation, Canada Foundation for Innovation, National Sciences and Engineering Research Council scholarship, Government of Alberta scholarship, Faculty of Medicine and Dentistry (UofA) scholarship

\section{Background}

Deep vein thrombosis (DVT) affects approximately 45,000 Canadians annually. Of specific concern are immobilized patients who cannot use anti-coagulants because of bleeding risks or compression devices due to discomfort. Intermittent electrical stimulation (IES) may present an alternative prophylactic intervention by activating the calf-muscle pump to increase venous return and prevent stasis. The objective of this study was to determine the required stimulation intensity to increase venous velocity in typical and mobility impaired subjects.

\section{Methods}

The study included three groups. The first group consisted of typical subjects $(n=12)$. Testing was performed on the right leg, stimulating the gastrocnemius and the tibialis anterior muscles sequentially. Gastrocnemius stimulation intensity was modulated to produce gradual increases in force. Doppler ultrasound was used to measure the baseline (before stimulation) and peak (during stimulation) popliteal venous velocities. Isometric ankle forces were measured using a custom-built apparatus. The second group consisted of typical subjects $(n=10)$; femoral and popliteal venous velocity were measured. The third group consisted of in-patients, post-stroke who were relatively immobile $(n=10)$; testing was performed on the more affected leg. Lastly, a questionnaire determined each subject's comfort.

\section{Results}

In typical subjects, a significance increase in popliteal venous velocity $(6.27 \pm 1.81 \mathrm{~cm} / \mathrm{s}$ versus $55.27 \pm 25.89 \mathrm{~cm} / \mathrm{s}, \mathrm{p}=0.014)$ and femoral venous velocity $(7.89 \pm 2.58 \mathrm{~cm} / \mathrm{s}$ versus $38.84 \pm 13.31 \mathrm{~cm} / \mathrm{s}$, $\mathrm{p}=0.002$ ) from baseline, was achieved at 11-20\% MVC. Post-stroke subjects showed an increase in popliteal venous velocity compared to baseline $(8.93 \pm 1.98 \mathrm{~cm} / \mathrm{s}$ versus $52.92 \pm 22.38 \mathrm{~cm} / \mathrm{s}, \mathrm{p}=0.013)$ at 51 $60 \%$ of the maximum recorded contraction with stimulation. The level of discomfort of the stimulation was rated "very little" to "moderate" in all subjects.

\section{Conclusion}

An increase in popliteal [all subjects] and femoral [typical subjects] venous velocities was achieved at comfortable levels. Further research is required in acute care settings to further determine the feasibility of IES as a prophylactic method for DVT. 


\section{Burnout and its Associated Factors Among Medical Residents: A Meta-Analysis and Meta-Regression}

\author{
Zahra Sohani $M D P h D^{1,2}$ and Leen Naji $M D^{3}$ \\ ${ }^{1}$ Faculty of Medicine, University of Toronto, Toronto, ON, \\ Canada \\ ${ }^{2}$ Department of Internal Medicine, McGill University, \\ Montreal, QC, Canada \\ ${ }^{3}$ Department of Family Medicine, McMaster University, \\ Hamilton, ON, Canada
}

\section{Background}

Burnout is increasingly recognized as a crisis in the medical profession, affecting both physician wellbeing and patient care. As resident physicians are tasked with tremendous responsibility whilst learning and integrating new skills, they are a particularly susceptible group.

\section{Objectives}

Our primarily aimed to establish the prevalence of burnout among medical residents based on a meta-analysis of global literature. Secondarily, we used this data to: 1) identify risk and protective factors for burnout, and 2) use meta-regression to establish whether prevalence in burnout varies by country of training, year of study, and specialty of training.

\section{Methodology}

We searched Medline, EMBASE, PsycINFO, Cochrane, Web of Science and ERIC from their inception to August 21, 2018. Burnout prevalence and study characteristics from 197 studies were extracted in duplicate by 5 investigators. Pooled prevalence was estimated using a random effects model with the restricted maximum-likelihood estimator. For our secondary analysis, we employed meta-regression using a random effects model.

\section{Results}

Our study encompasses data collected over 30 years among 44,000 residents across 47 countries. We found the pooled global prevalence of burnout to be $47.3 \%$ (95\% CI $43.1 \%$; 51.5\%). Four datasets studied Canadian residents and had a burnout prevalence of $39.5 \%$. Among studies included in our review, a majority found depression, stress, and lower job satisfaction to be associated with higher rates of burnout. Our analysis also uncovered three novel findings: we report that despite changes in duty hours, the prevalence of burnout has not changed over the past 2 decades. Burnout appears to vary by region, with residents of European countries experiencing the lowest level. Lastly, we found burnout to be unaffected by specialty of practice.

\section{Conclusions}

We establish a prevalence of burnout at $47 \%$ among resident physicians. Our findings suggest that systemic factors have an important role in resident wellness.

\section{"R1 On-Call Nightmares": A Collaborative Multi-Residency Curriculum to Prepare Junior Residents for Medical Emergencies}

Jordan Sugarman ${ }^{1}$, Erica McKenzie ${ }^{2}$, Tim Chaplin ${ }^{3}$, Ghazwan Altabbaa ${ }^{1}$

${ }^{1}$ Department of Medicine, University of Calgary, Calgary, Alberta

${ }^{2}$ Department of Clinical Neurosciences, University of Calgary, Calgary, Alberta

${ }^{3}$ Department of Emergency Medicine, Kingston Health Sciences Centre, Kingston, Ontario

\section{Background}

First-year physicians-in-training feel ill-prepared for and anxious about responding to medical emergencies. The opportunity for residents to practice the assessment and management of acute inpatient medical situations in a simulated learning environment is a valuable adjunct to training and facilitates increased resident comfort and patient safety on the wards. $2 \mathrm{~A}$ high-fidelity simulation course aimed at improving comfort with medical emergencies has already been successfully implemented at Queen's University in Kingston, Ontario.2 Peer-reviewed data demonstrates this course to be a cost-effective way of reinforcing essential skills and increasing comfort and competency at managing acute medical issues. Here, we discuss the design and deployment of a similar curriculum at the University of Calgary.

\section{Methods}

A four-track half-day simulation course entitled "R1 On-Call Nightmares", followed by a 30-minute debriefing session, was piloted in August 2019. Participants included first-year postgraduate medical residents from the Internal Medicine and Neurology residency programs. Participants were administered a 5-point Likert scale post-participation survey. Quantitative measures were aggregated using traditional statistical methods and standard errors of the mean were calculated. Narrative feedback was collected and aggregated in a two-question post-participation questionnaire.

\section{Results}

Forty first-year residents from the Internal Medicine and Neurology residency programs participated in the course. Scenarios were 
felt to be relevant and realistic to actual ward emergencies (average 5-point Likert response $4.75+/$ - 0.037), with participants reporting high awareness of overnight resources available in a medical emergency after the course $(4.58+/-0.081)$. The course was highly recommended for future first-year residents $(4.89+/$ $0.050)$. The cost of the course was $\$ 25$ per resident participant.

\section{Conclusions}

The "R1 On-Call Nightmares" course is a safe, cost-effective and highly-recommended intervention in addressing resident discomfort with medical emergencies. Future directions include the addition of two new scenarios and expanding the delivery in collaboration with other residency programs.

\section{Clinical Teaching Unit Design: A Systematic Review of Evidence-Based Practices for Clinical Education and Health Service Delivery}

Brandon Tang MD

$M S c^{1}$, Katrina Dutkiewicz $M D^{2}$, Stephan Saad

$M D^{3}$, Jocelyn Chai ${ }^{4}$, Kristin Dawson $\mathrm{PhD}^{4}$, Ryan

Sandarage4, Vanessa Kitchin ${ }^{5}$, Iain McCormick MD

$B A H^{2}$, Barry Kassen MD FRCP FACP MACP

${ }^{1}$ Department of Medicine, University of British Columbia, British Columbia, Canada

${ }^{2}$ Division of General Internal Medicine, University of British Columbia, British Columbia, Canada

${ }^{3}$ Division of Infectious Diseases, University of British Columbia, British Columbia, Canada

${ }^{4}$ Faculty of Medicine, University of British Columbia, British Columbia, Canada

${ }^{5}$ Vancouver Fraser Medical Program and Woodward Library, University of British Columbia, British Columbia, Canada

${ }^{6}$ Clinical Teaching Unit Director and Acting Head of the Division of Community General Internal Medicine, Division of General Internal Medicine, University of British Columbia, British Columbia, Canada

Funding: This work was supported by the Department of Medicine at St. Paul's Hospital in Vancouver, British Columbia, Canada

\section{Background}

The Clinical Teaching Unit (CTU) has emerged as a near ubiquitous model of clinical education across Canadian and international medical schools, since it was first proposed over 50 years ago. However, health care has changed dramatically over this period, and we thus aimed to review existing evidence on principles of CTU design that optimize clinical education and health service delivery in the 21 st century.

\section{Methods}

We performed a systematic review in accordance with the Cochrane Review protocol. Databases, including MEDLINE, Embase, Cochrane Database of Systematic Reviews and CINAHL, were searched to find primary research articles published from 1993 to 2019 which discussed trainee education and/or health care delivery in context of a clinical teaching unit or other teaching ward.

\section{Results}

After deduplication, our search yielded 1938 potentially relevant articles which are currently undergoing a two-step screening process of abstract and full-text review. Studies which met inclusion criteria after full-text review were analyzed according to the Star Model which views health systems as a collection of interdependent subsystems including: strategy, structure, human resources, incentives, and information and decision support. We added a sixth subsystem for education given its core relevance to CTUs. Initial results suggest that most existing evidence focuses on the structure and education subsystems, including approaches to ward rounds, optimal call structures, and novel educational strategies. However, the majority of articles involved low to moderate quality evidence as per the GRADE system.

\section{Conclusion}

Efforts should be made to generate higher quality evidence on the design of clinical teaching units as a dual model for both clinical education and health service delivery. In addition, further knowledge translation efforts may be necessary to ensure that known best practice in CTU design becomes common practice.

Keywords: Clinical teaching unit, systematic review, medical education, evidence-based education, health service delivery Best Research Poster - Dr. Erin Helson 


\section{Rocky Mountain ACP/AMA Internal Medicine - Banff}

\section{Case Study Abstracts}

\begin{abstract}
The winners were as follows:
\end{abstract}
Best Abstract, Clinical Case - Dr. Brandon Tang

Best Abstract, Original Research - Dr. Zahra Sohani and Dr. Leen Naji

Best Clinical Case Poster - Dr. Victoria Nkunu

Best Research Poster - Dr. Erin Helson

\section{Hamman's Sign: A Precordial Sound Leading to Diagnosis of Spontaneous Pneumomediastinum from Labour And Delivery}

Contributing author: Dr. Lillian Chan (Obstetric Medicine and GIM Fellow; University of Alberta)

No funding sources.

Supervising Faculty: Dr. Jordan Marit

\section{Background}

Spontaneous pneumomediastinum is uncommon. It often presents with chest pain, dyspnea, and swelling or subcutaneous emphysema. Hamman's sign is an auscultatory clue that suggests pneumomediastinum.

\section{Case Description}

A healthy 24-year-old primiparous woman at 41 weeks and 5 days' gestation developed a generalized tonic-clonic seizure immediately after delivery. She had a prolonged second stage of labour that lasted 4.5 hours. Post-seizure, her main symptom was pharyngeal pain. On exam, a loud, systolic scratching noise was auscultated over the left sternal border. Oxygen saturation was $97 \%$ on 2 liters of oxygen. The remainder of her vital signs and physical examination was normal. The abnormal sound prompted urgent chest imaging. A chest X-ray and subsequent CT chest demonstrated a moderate amount of pneumomediastinum and a small left pneumothorax. There was emphysema in the retropharyngeal soft tissues. A trace amount of intraperitoneal air was noted, due to air tracking from the mediastinum. There was no evidence of esophageal perforation on CT. A CT and MRI brain were normal. Lab investigations were significant only for hyponatremia at $125 \mathrm{mmol} / \mathrm{L}$ that resolved spontaneously, and elevated serum lactate and CK levels secondary to seizure activity. There were no convincing signs or symptoms to suggest eclampsia as the cause of her seizure. After consultation with thoracic surgery, she was managed conservatively with oxygen therapy and serial chest imaging until resolution.

\section{Conclusion}

Spontaneous pneumomediastinum is a rare complication with a reported incidence of less than 1:44 000, and even lower at 1:100 000 in pregnancy or labour. It is likely underdiagnosed, and can occur in situations of prolonged Valsalva, such as hyperemesis gravidarum or diabetic ketoacidosis, coughing, and labour. Hamman's sign, a systolic crunching noise caused by movement of the heart amid mediastinal emphysema, may be a useful diagnostic clue for pneumomediastinum.

\section{A Mysterious Case of Night Sweats \\ C. Collins and L. Bridgland}

A previously healthy 59-year-old woman was admitted to Internal Medicine with a 2-month history of night sweats, fevers, polyarthralgias, hallucinations (auditory/visual), weight loss, and perioral vesicular lesions. These symptoms started after a trip to Mexico. Investigations revealed elevated inflammatory markers with new onset anemia (hemoglobin 89). A lumbar puncture showed an elevated WBC count of 31 (predominantly lymphocytes) with a negative viral panel.

A CT head and MRI brain were normal. Extensive infectious work up was negative. She was treated with intravenous acyclovir for presumed HSV encephalitis. Her symptoms resolved in hospital.

Given her constitutional symptoms, a CT chest/abdomen/ pelvis was done which revealed a solitary pulmonary nodule and no lymphadenopathy. She, therefore, underwent a PET CT that showed metabolic activity within the ascending colon.

Subsequent gastroscopy, colonoscopy and CT enterography were inconclusive. She had recurrence of fevers and night sweats following discharge. Inflammatory markers remained elevated. 
A bone marrow biopsy showed atypical lymphoid aggregates suspicious for $\mathrm{B}$ cell lymphoma. The decision at lymphoma rounds was to monitor clinically. A repeat CT chest showed extensive patchy consolidation throughout both lungs. Although she had no respiratory symptoms, she had bronchoscopy, which was unremarkable. A repeat PET CT revealed a pattern suggestive of organizing pneumonia. Open lung biopsy revealed foci of organizing pneumonia and non-necrotizing granulomas. Initial AFB stain was positive, but the culture was negative.

A diagnosis of organizing pneumonia (OP) was made based on radiographic and histopathologic features. Asymptomatic OP occurs in up to $14 \%$ of patients. OP can be idiopathic, secondary to infection, or occur in a specific context (e.g., rheumatoid arthritis). In our case, HSV or gastrointestinal illness were hypothesized to be the cause. Treatment generally consists of corticosteroids; however, in our patient treatment was deferred while awaiting AFB culture. Symptoms and laboratory abnormalities resolved without treatment.

\section{Diagnosis of Central Diabetes Insipidus in a Patient with Acute Myeloid Leukemia}

Victoria Nkunu, MD, Justin Smith, MD, Lauren Bolster, $M D, M E d, F R C P C$

Division of Hematology, Faculty of Medicine, University of Alberta, Edmonton, Canada

\section{Introduction}

Central diabetes insipidus (DI) is a rare clinical manifestation of acute myeloid leukemia (AML), primarily described in case reports within the medical literature. While a distinct pathophysiology linking central DI and AML is unknown, their relationship has important implications for diagnosis and prognostication of AML.

\section{Case Descriptions}

A thirty-two-year-old woman was admitted after a complete blood count revealed anemia, leukocytosis and 69\% circulating blasts. History revealed six-months of constitutional symptoms, polydipsia, and polyuria. Bone marrow biopsy confirmed AML with abnormal cytogenetics $(45, \mathrm{XX},-7, \operatorname{inv}(3))$. The patient's initial sodium level was normal at $140 \mathrm{mmol} / \mathrm{L}$. After depriving oral intake for an abdominal ultrasound, she developed hypernatremia (155 $\mathrm{mmol} / \mathrm{L}$ ), with a concurrent low urine osmolality of $141 \mathrm{mmol} /$ $\mathrm{kg}$. Magnetic resonance imaging of the sella turcica demonstrated thickening of the pituitary stalk with absence of the posterior pituitary bright spot, suggesting leukemic involvement of the CNS with secondary diabetes insipidus, despite initial CSF analysis being negative for blasts. The patient received oral desmopressin
(DDAVP) which normalized serum sodium levels, increased urine osmolality, and improved her symptoms of DI. She was treated with induction chemotherapy (IDAC), however repeat bone marrow done at day 17 for intractable bone pain demonstrated refractory leukemia and she is presently receiving re-induction chemotherapy (NOVE-HDAC).

\section{Discussion}

Symptoms of DI (polyuria and polydipsia) may precede initial diagnosis of AML. In a minority, DI is diagnosed in patients with AML in remission, developing prior to their leukemia relapse. Electrolyte workup and response to DDAVP therapy confirms the diagnosis of central DI. On MRI, there is often pathology detected in the pituitary stalk, but often no evidence on either CSF analysis or autopsy of leukemic infiltration of the pituitary. Unfortunately, these patients tend to have aggressive leukemia with most responding poorly to chemotherapy, and a high mortality rate due to refractory leukemia.

\section{A Case of Streptococcus Canis Bacteremia, Osteomyelitis, Sacroiliitis, Myositis, and Abscess}

Allyshia Van $\mathrm{Tol}^{1}$, Brandon Tang MD MSc', Iain Mackie MD FRCPC ${ }^{3}$

${ }^{1}$ Faculty of Medicine, University of British Columbia, British Columbia, Canada

${ }^{2}$ Department of Medicine, University of British Columbia, British Columbia, Canada

${ }^{3}$ Division of General Internal Medicine, Vancouver General Hospital and University of British Columbia, British Columbia, Canada

Funding: No sources of funding to report.

A previously healthy 26-year-old male presented to the emergency department with a 2-day history of erythema, pain, and swelling of the left foot, consistent with acute cellulitis. The patient was initially discharged home on oral cephalexin, but later recalled after $2 / 2$ blood cultures grew gram positive cocci. Blood cultures speciated as Streptococcus canis, a group G beta-hemolytic Streptococcus species which resides on the skin and mucosal surfaces of dogs.

History was unremarkable save for a 2-week history of lower back pain precipitated by a wrestling injury. The patient had occasional contact with a friend's dog (species unknown), but there was no history of traumatic injury to the foot, canine bite, or scratch wound. Given the subacute back pain, CT spine was obtained which demonstrated possible right piriformis myositis and S1 osteomyelitis. These findings were confirmed on 
a subsequent MRI, which additionally demonstrated right erector spinae myositis, right sacroiliitis, and multiple collections in the right posterior paraspinal soft tissues (largest $25 \mathrm{x}$ $19 \mathrm{~mm})$. Transthoracic echocardiogram did not demonstrate valvular vegetations. The $\mathrm{S}$. canis was pan-sensitive and the patient was eventually discharged home on penicillin $G$ to complete a 6-week course of IV antibiotics.

S. canis rarely causes infection in humans, however, it can manifest in many different forms when it does. According to the literature, these include skin and soft tissue infections $(n=39$; $57 \%)$, bacteremia $(n=17 ; 25 \%)$, osteoarticular infections $(n=4$; $6 \%)$, and endocarditis $(n=3 ; 4 \%)$. A clear portal of entry, such as a break in the skin, is not necessary for infection. We suggest that management of $\mathrm{S}$. canis bacteremia should involve screening for sites of metastatic infection in addition to infectious diseases consultation. However, despite its propensity for systemic involvement, $\mathrm{S}$. canis is often susceptible to narrow spectrum antibiotics including penicillin.

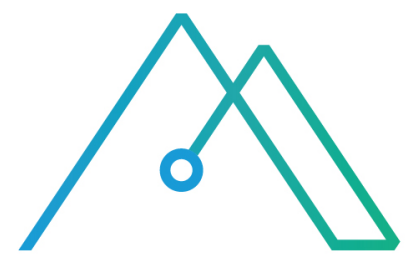

\section{ROCKY MOUNTAIN \\ Internal Medicine}

